\title{
COMMISSION 31: TIME
}

\author{
(L'HEURE)
}

\section{PRESIDENT: T. Fukushima}

\section{VICE PRESIDENT: G. Petit}

ORGA NIZING COMMITTEE: D.C. Backer, G. Beutler, V.A. Brumberg, H.F. Fliegel, S.M. Leschiutta, J. Luck, P. Paquet, E. Provebio, G.R. Qi, C. Thomas, C. Veillet, G.M.R. Winkler \& Z.C. Zhai

\section{CCTF RECOMMENDATIONS}

The CCTF (formerly named CCDS) held its 14th meeting on 20-22 April 1999. Following the discussions, seven Recommendations were adopted and submitted to the Comité International des Poids et Mesures (CIPM). The list is the following:

1. Recommendation S 1 (1999): Mise-en-pratique of the definition of the second.

2. Recommendation S 2 (1999): On stating uncertainty in comparisons involving primary frequency standards.

3. Recommendation S 3 (1999): On the comparison of primary frequency standards.

4. Recommendation S 4 (1999): On the use of multi-channel and multi-code GPS and GLONASS time receivers.

5. Recommendation S 5 (1999): Time and frequency comparisons using GPS phase and code measurements.

6. Recommendation S 6 (1999): Future global navigation satellite systems.

7. Recommendation $S 7$ (1999): On Two-Way Satellite Time and Frequency Transfer

Additionally discussed were the present form of UTC and mostly the interest of preserving the leap second. The advantages and disadvantages of several options regarding the future use of leap seconds were compared. The CCTF, however, felt that it did not have the authority to propose any action. Then CCTF decided to ask the BIPM Director to write to the relating international bodies including IAU so as to draw their attentions to this issue while recommending the usage of TAI in case a time scale without discontinuity is needed. Also it was decided, in order to make more expedite the process, to ask the opinions of the various Commissions of the Scientific Unions. 


\section{COMPUTATION AND DISSEMINATION OF TAI AND UTC}

Since 1996, the quality of the timing data used for the computation of TAI has further improved. Around $75 \%$ of the clocks are either commercial Cs clocks of the new type or active, auto-tuned $\mathrm{H}$ masers, and they contribute $89 \%$ of the total weight of TAI. The TAI algorithm has been modified twice. Since 1st of January 1996, TAI and UTC are calculated for all modified julian dates ending in 4 and 9 (one point every 5 days). Since 1st of January 1998 , the weighting method has been changed by adopting a relative maximum weight of a clock, and the calculation interval of TAI has been reduced from two to one month. Introduction of new primary frequency standards has also improved the accuracy of TAI. Most of these are "classical" Cs beam standards, some of them using optical techniques for the excitation and detection of the atoms. The first results of a primary standard using cold atoms in a fountain geometry have been submitted in 1995. This is a first step towards a larger number and larger diversity of primary frequency standards. The efficiency and reliability of the calculation and diffusion of TAI and UTC are continuously kept under review. Some automatisation of the processes involved, taking advantage of the recent developments in electronic communication and data treatment facilities at BIPM, should help ensuring these goals.

The GPS, operated from Schriever Air Force Base in Colorado Springs, Colorado, USA, is being kept more closely synchronized with UTC by a new installation there called the USNO Alternate Master Clock (AMC). This installation contains $3 \mathrm{H}$ masers, a dozen Cs standards, and several GPS receivers. The AMC's master clock provides a frequency which is used with about $28 \%$ of the weight in the computation of GPS time. The AMC's master clock also serves as a back-up for and integrity check on the USNO Master Clock in Washington, D.C., to which it is kept synchronized to within 1 ns by a two-way satellite link. USNO additionally operates a two-way link with a site in Bahrain over a $10983 \mathrm{~km}$ baseline, the longest in operation. Time transfer accuracies within 3 ns are routinely achieved. GPS time transfer is increasingly being done with multi-channel receivers whose averaging over several satellites has, for instance, allowed USNO to achieve precisions of less than $1 \mathrm{~ns}$ over 1 day for a $2400 \mathrm{~km}$ baseline. USNO has been conducting precise time and frequency transfer experiments using GPS carrier phase.

Remote computers and networks can obtain millisecond sychronization with UTC over the Internet using Network Time Protocol (NTP) servers synchronized with national timing centers such as USNO.

The Major T\&F institutes in Asian-Pacific Region are constructing a TWSTT network in this region (CRL, NML, CSAO, and NRLM) under mutual cooperation. Main tools for this TWSTT network are the Time transfer modem, TWT-100, and the Earth station with a $1.8 \mathrm{~m}$ dish $\mathrm{Ku}$-band antenna. Also CRL has decided to shift the radio frequency band from HF to LF for time and frequency service in Japan. To perform this direction, CRL has started constructing new LF stations in Japan. The construction of Otakadoyayama Station, one of new LF stations, has completed and the station has been operational since June 10,1999 . This station emits the center frequency of $40 \mathrm{kHz}$ and the radiation power more than $10 \mathrm{~kW}$. The call sign of station is JJY. The construction of the second LF station has been started in 1999 and it will be operational around middle of 2001.

\section{RAPID DISSEMINATION OF LOD AND UT1}

The IERS Sub-bureau for Rapid Service and Predictions of Earth orientation parameters (EOPs) combines contributed analysis results from a variety of techniques and observing groups to form a homogeneous daily time series which is updated daily and distributed twice weekly as "IERS Bulletin A". In addition to polar motion, IERS Bulletin A has become increasingly reliant on GPS-based estimates for length of day (LOD). The IGS started producing an official LOD product on 2 March 1997 using a weighted combination of LOD results submitted by each IGS Analysis Center (AC). To calibrate for LOD biases, each series is compared with the most recent 21 days of non-predicted UT1 values from Bulletin 
A (Kouba and Mireault, 1997; Ray, 1996). Shortly after its advent, the IGS combined LOD results were introduced into the Bulletin A combination to extend the UT1 value of the most recent VLBI determination forward by integration. A few months later an independent set of GPS-based estimates of universal time, derived at USNO and described below, were also included in Bulletin A. About three weeks of the most recent estimates are used, after calibration in offset and rate compared to overlapping UT1 results from VLBI. In April 1998, an analogous universal time series from the EMR AC was added in a similar manner. The EMR analysis strategy differs from the other IGS groups in applying a priori orbit constraints that allow both universal time and LOD to be estimated simultaneously. These three series together have proven very successful in extending UT1 results forward from the latest VLBI determinations, which can have a latency of up to about a week. As a consequence, the last non-predicted UT1 value in Bulletin A is now generally more accurate than 100 microseconds, usually considerably more so.

For real-time users, given two updates of Bulletin A each week, the longest prediction interval is 7 days. This means that real-time users can experience polar motion prediction errors up to $\sim 2.4$ mas and UT1 errors as large as $\sim 1 \mathrm{~ms}$. Predictions of UT1 variation are more problematic because the geophysical excitation is about an order of magnitude larger than for polar motion. To reduce these prediction errors significantly requires more frequent Bulletin A updates, preferably done daily shortly after the IGS Rapid products are released. Such a process was implemented during 1998 and reduces the longest prediction interval from 7 days to $\sim 58$ hours, under normal circumstances. Now the maximum errors for real-time users should be less than $\sim 0.7$ mas for polar motion and less than $\sim 0.2 \mathrm{~ms}$ for UT1. Results are available at http://maia.usno.navy.mil/.

\section{IGS/BIPM TIME AND FREQUENCY PROJECT}

The "IGS/BIPM Pilot Project to Study Accurate Time and Frequency Comparisons using GPS Phase and Code Measurements" is sponsored jointly by the IGS and BIPM. A working group was formed in spring 1998 which first met at BIPM in June. The central goal is to investigate and develop operational strategies to exploit GPS measurements for improved availability of accurate time and frequency comparisons worldwide. This will become especially significant for maintaining the international UTC timescale as a new generation of frequency standards emerges. The respective roles of the IGS and BIPM are complementary and mutually beneficial. The IGS and its collaborating participants bring a global GPS tracking network, an efficient data delivery system and state-of-the-art data analysis groups, methods and products. The BIPM and its collaborating timing laboratories contribute expertise in high-accuracy time and frequency measurements, calibration methods, time scale algorithms, and the formation and dissemination of UTC. Two meetings were organized in 1998, on 22-23 June at the BIPM, and on 30 November associated with the 30th PTTI meeting. Activities generally fall into the following areas:

- Deployment of GPS receivers: The IGS network currently consists of about 200 permanent, continuously operating stations globally distributed. Of these, external frequency standards are used at: $\sim 30$ with H-masers, $\sim 20$ with cesium clocks, and $\sim 20$ with rubidium clocks; the remainder use internal crystal oscillators. Currently, 11 IGS stations are located at timing laboratories, of which four also participate in two-way satellite time transfer operations.

- GPS data analysis: Of the IGS Analysis Centers, all but two already provide satellite clock estimates. A plan has been adopted to expand the IGS products during 1999 to include combined receiver clocks. To improve the timescale alignment and weighting of Analysis Center solutions, a set of "fiducial clock" sites was adopted, most of which are equipped with $\mathrm{H}$-maser frequency standards.

- Instrumental delays: Efforts are underway to develop techniques for measuring the calibration biases which relate internal receiver clocks to external time standards. 
When available for IGS stations located at timing laboratories, tracability to UTC can be established for IGS clock products. This effort is the foremost technical challenge facing the Pilot Project.

- Comparison experiments: So far, only a few controlled experiments have been conducted to compare geodetic timing results with simultaneous, independent techniques. However, high-quality frequency comparisons already appear entirely feasible provided that reasonable care is taken to minimize environmentally induced variations.

For further information, please refer to the Web site http://maia.usno.navy.mil/gpst.html.

Also the work includes the involvement from the IGS Central Bureau (IGS/CB), which is located at NASA/JPL. The IGS/CB is the executive arm of the IGS, and as such provides enabling support to the Pilot Project by participating in development and dissemination of guidelines of GPS station operation for time transfer, incorporating time transfer activities into its network coordination task, and providing meeting and publication support.

\section{OTHER TOPICS}

- The activity of the BIPM/IAU Joint Committee on relativity for space-time reference systems and metrology (JCR) is shown in its Web site: http://www.bipm.fr/WG/CCTF/JCR/.

- A. Irwin and T. Fukushima (1999) recomputed the time ephemeris, the locationindependent part of TCB and TCG, and gave better estimates of $L_{C}$ and $L_{B}$.

- P. Kammeyer (1999) developed a method to determine UT1-like variations from an analysis of GPS orbit planes as estimated for IGS Rapid submissions.

- USNO has begun the construction of a cesium fountain frequency standard.

- The time and frequency standards maintained at JPL are used in applications ranging from communications and spacecraft navigation, to support radio science and physics experiments.

- CRL and NIST collaborated on the development of the optically pumped cesium primary frequency standard CRL-O1 since January 1996. Its achieved total uncertainty was $2.4 \times 10^{-14}$.

- NRLM modified the optically pumped cesium frequency standard, NRLM-4. Its frequency stability is $5 \times 10^{-13} T^{-1 / 2}$, and developed a Cs atomic fountain frequency standard system aiming an accuracy of $1 \times 10^{-16}$.

- CRL has conducted the pulsar timing observation of PSR1937+21 once a week for more than one year and obtained about 2-3 microsecond of receiving precision using $\mathrm{S}$-band receiver of Kashima $34 \mathrm{~m}$ antenna. 